BMJ Open

Sport \&

Exercise

Medicine

\title{
Patterns of platelet-rich plasma use among Australasian sports physicians
}

\author{
David J Samra, ${ }^{1,2}$ John W Orchard ${ }^{1,3}$
}

To cite: Samra DJ, Orchard JW. Patterns of platelet-rich plasma use among Australasian sports physicians. BMJ Open Sport Exerc Med 2015;1:e000054. doi:10.1136/bmjsem-2015000054

- Prepublication history and additional material is available. To view please visit the journal (http://dx.doi.org/ 10.1136/bmjsem-2015000054).

Received 18 August 2015 Accepted 17 October 2015

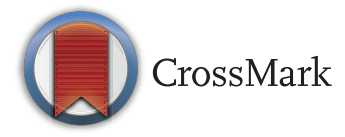

${ }^{1}$ The Sports Clinic, University of Sydney, Sydney, Australia ${ }^{2}$ Faculty of Health Sciences, University of Sydney, Sydney, Australia

${ }^{3}$ School of Public Health, University of Sydney, Sydney, Australia

Correspondence to David J Samra;

davidjsamra@gmail.com

\section{ABSTRACT}

Background: We hypothesised that the application, production and administration of platelet-rich plasma (PRP) varies widely among sports physicians, bringing into question the validity and consistency of PRP described in research and clinical use. We also assessed congruence between the reported clinical indications for PRP, and the available research evidence for these indications.

Methods: We conducted an anonymous 23 question online survey of 153 current Fellows of the Australasian College of Sports Physicians (ACSP), using an emailed link. It was opened from April 2014 until August 2014.

Results: The survey confirmed that there is wide variation in the application, production and administration of PRP. Over one-third (38\%) of sports physicians performed PRP injections themselves. Almost half of clinicians (49\%) did not provide the service themselves, and only referred for PRP injections. The remaining clinicians did not inject PRP or refer for PRP injections at all. Clinicians who provided PRP injections varied from an average of 0-500 injections per month, with a median of 12 times per month. Australian sports physicians were far more likely to use PRP than their New Zealand counterparts. For sports physicians who provided or referred for PRP injections, tendinopathy was overwhelmingly cited $(n=63)$ as the condition for which clinicians thought PRP was most effective. 30 respondents cited effectiveness for osteoarthritis.

Conclusions: This study confirms that there is no clear consensus among sports physicians on the preparation, administration or best clinical indications for PRP.

\section{INTRODUCTION}

Sport and exercise medicine (SEM) physicians may use platelet-rich plasma (PRP) injections as a therapeutic adjunct in the management of difficult musculoskeletal problems. However, despite a compelling theoretical rationale and promising preclinical research, it is considered an unproven therapy that does not seem to have universal support among SEM physicians. ${ }^{1}{ }^{2}$ Laudy $e t a l^{3}$ indicate that there is a lack of consistent high-quality research evidence, a lack of consistency in the formulation of PRP produced

\section{What is known}

There is very little high quality evidence to support the application of platelet-rich plasma (PRP) for musculoskeletal problems other than mild to moderate knee osteoarthritis.

\section{What this study adds}

- The threshold, indications, preparation and administration of PRP use differ widely among sport and exercise medicine (SEM) physicians. The lack of standardisation in methodology for PRP preparation creates uncertainty in the generalisability of research conclusions - perhaps perpetuating the beliefs that PRP may still be effective when applied with as yet unknown, 'optimal' methodology.

- SEM clinicians are polarised in their attitudes towards the place of PRP as an evidence-based therapeutic agent in patient care.

- Despite high-quality research showing low, if not absent benefit over placebo for PRP on wide range of clinical problems, many clinicians express support from clinical experience.

by clinicians, and varied clinical indications between clinicians for adopting its use; and clearly these factors have influenced the way that PRP is viewed by SEM physicians and their peers. The current study aimed to assess the practices and beliefs of SEM physicians in relation to PRP as an injectable therapy, and to correlate this with the best available research on PRP for musculoskeletal problems. Identifying the discrepancies between clinical research and real world practice is important to guide, validate and standardise future research and practice in this field.

The process of procuring PRP lends itself to inconsistency both between clinicians and for the same clinician. The process involves venesection (varying quantities and tubes), centrifugation of peripheral blood (varying force, speed and time) and aspiration of a platelet concentrate (varying needle gauge, aspiration technique and size of platelet-rich 
zone of plasma). It may or may not involve variation in the number of centrifuges (single or double spin), or the use of activation methods (addition of mechanical disturbance, UV light, thrombin or calcium chloride). ${ }^{4}$ These permutations create a wide variability in the product and this creates uncertainty for researchers and clinicians as to what the optimal preparation method and formulation may be. ${ }^{5}$ Commercial kits exist that appear to increase the consistency of this process, however, there is no evidence that the finished product is actually more consistent or effective than a simple centrifuge method using citrate tubes. ${ }^{6}$ Furthermore, the injection of PRP may be performed with or without ultrasound guidance to confirm anatomical accuracy. We aimed to assess the degree of consistency among SEM physicians in the production and application of PRP.

There is no agreement among SEM physicians on clinical indications for which PRP injections are considered effective. This is despite a wide range of musculoskeletal problems that are treated with PRP; from chronic tendinopathies, osteoarthritis of large and small joints, ligament injuries and even spinal pathologies. ${ }^{7}$ However, there have been very few high-quality trials to support any of these indications apart from mild-moderate severity knee osteoarthritis. ${ }^{8} 9$ PRP is an autologous product not requiring any regulation, preclinical testing or FDA approval. ${ }^{10}{ }^{11}$ Hence, clinical practice has preceded clinical trials; and extrapolation and appropriation of clinical evidence (of varying quality) have lead to highly diverse applications among different clinicians with differing expertise. With the lack of research supporting PRP, clinical decisions to use it therapeutically must be driven by other factors including clinician experience and patient preference. By virtue of their role as referral-based specialists in treating patients with more recalcitrant problems, SEM physicians may be more inclined to offer patients a PRP injection or referral for PRP injection. Hence, we also aimed to assess the degree of agreement among SEM physicians towards clinical indications for PRP.

\section{METHODS}

A simple 23-point multiple choice and free-text questionnaire (see online supplementary appendix A) was distributed via email to 153 Fellows of the Australasian College of Sports Physicians (FACSP), with the approval of the ACSP Research Committee and Sydney University Human Research Ethics Committee. The email inviting participants to participate included an online Internet link to the survey. The ACSP is the professional body representing SEM physicians in Australia and New Zealand. Fellows of the ACSP are medical practitioners who have completed all the requirements of specialist training and have been admitted to Fellowship by the ACSP Council.

The online submission was closed 4 months after the original email was sent. Four reminder emails were sent

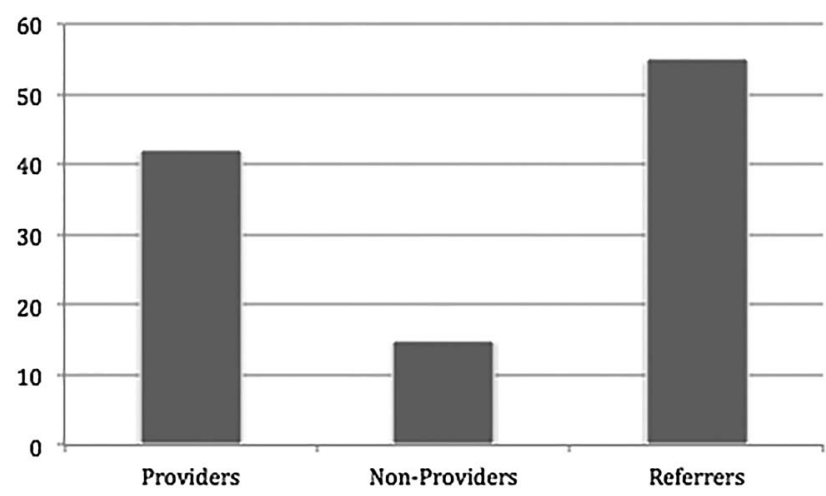

Figure 1 Number of sports physicians by providers and non-providers and referrers for platelet-rich plasma.

to Fellows to maximise the response rate. Once the survey was closed in August 2014, raw data were accessed and analysed using simple descriptive statistical techniques on SPSS V.22.

\section{RESULTS}

One hundred and fifty-three FACSPs were sent an email invitation to complete the survey and 112 responses were received; giving a response rate of $73 \%$. Of these, 97 responses were complete; giving a completion rate of $87 \%$.

\section{PRP providers, non-providers and referrers}

We found that most clinicians did not provide PRP themselves. Forty-two of the responding SEM physicians $(38 \%)$ reported providing PRP injections for their patients (PRP providers). Fifteen (13\%) did not provide PRP or refer for PRP injections (PRP non-providers), leaving 55 clinicians $(49 \%)$ who referred for PRP injections (PRP referrers), without applying it themselves (figure 1). Clinicians who performed their own PRP injections varied from an average of 0-500 injections per month, with a mean of 24 injections per month (and a median of 12 times per month). Australian clinicians were far more likely to provide PRP injections than their New Zealand counterparts $(\mathrm{p}=001)$. Almost half $(45 \%)$ of Australian clinicians provided PRP injections, compared with $6 \%$ of New Zealand clinicians (figure 2). Many respondents referred patients to radiologists for PRP injections (64\%), even if they themselves did not provide PRP. There was no significant association between the provision of PRP injections and the number of years in clinical practice $(\mathrm{p}=0.173)$.

\section{Indications for PRP}

For clinicians who provided or referred for PRP injections, various tendinopathies were mentioned by $56 \%$ of respondents $(n=63)$ as the conditions for which clinicians thought PRP was most effective, from their experience. Respondents were allowed to cite up to three conditions. Common extensor origin tendinosis $(n=30)$, hamstring origin tendinopathy $(\mathrm{n}=17)$ and patella tendinopathy $(n=17)$ were the most frequent tendinopathies 


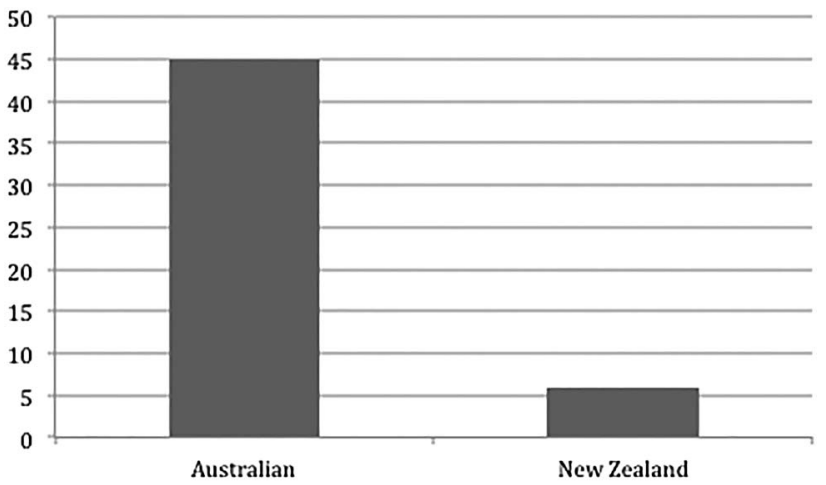

Figure 2 Percentage of Australian versus New Zealand providers of platelet-rich plasma.

named. Thirty respondents $(27 \%)$ reported clinical experience of success with PRP for osteoarthritis (most specifying osteoarthritis of the knee).

\section{Preparation and administration of PRP}

Forty per cent of clinicians who performed PRP injections themselves used commercial kits. Five different commercial kits were referenced: Terumo, Regen, ACP, Magellan and Biomet from most-to-least popular. Reasons for use included ease of asepsis, volume of product and reproducibility. However, more than half of those who used commercial kits $(21.5 \%$ of all PRP users) also used simple centrifuge methods (depending mainly on the content of the PRP they aimed to produce, practice facilities and patient preference). Of those who used a simple centrifuge and citrate tubes to produce PRP, the majority $(59.5 \%)$ cited reduced cost as a factor, while $47 \%$ thought there was not enough evidence that commercial kits are superior. Almost one-third $(28.5 \%)$ of clinicians who provided PRP injections used activating techniques (mechanical, thrombin or calcium chloride). Two-thirds $(67 \%)$ of clinicians using PRP said they usually used ultrasound guidance for their PRP injections, most $(87 \%)$ citing injection accuracy and efficacy as the reason for using guidance.

\section{DISCUSSION}

PRP injections are still considered a novel therapy after over 15 years of use in SEM. Quality research evidence has been well preceded by delivery to patients, which is a unique characteristic of this off-label therapy. As an autologous product there have been no rigorous tests of safety and efficacy prior to application on patients, as one would expect with any pharmaceutical product. ${ }^{11}$ The final product and its effectiveness are userdependent (varies both between and within clinicians) and patient-dependent (varies between patients since it is autologous). ${ }^{12}$ There are many variables in that are not controlled between users (in either the clinical or research environment), and it is impossible to standardise all of the multiple steps of this intervention from blood collection to injection. It is an example of innovation in medicine being driven by patient demand and perceived benefits over and above concrete evidence of efficacy.

The current state of clinical evidence for PRP is sobering in comparison to promising preclinical trials. The experience of many sports physicians surveyed in this study is that PRP is effective as a treatment option for tendinopathy, which contrasts with the highest quality clinical evidence. PRP appears to have only short-term (12-week) benefit for patella tendinopathy, ${ }^{13}$ no benefit above saline injections for chronic Achilles tendinopathy, ${ }^{14}$ and no benefit above saline injections for chronic lateral epicondylar tendinopathy. ${ }^{15}$ While PRP is probably not detrimental to healing, as corticosteroid injections are now thought to be, ${ }^{16}$ most RCTs with a low risk of bias show lack of effectiveness for tendinopathy when compared with the normal saline placebo. ${ }^{5}$ On the other hand, there is moderate evidence of small improvements in pain and function in early knee OA. ${ }^{3} 17$ The pertinent RCTs are limited by their generally high risk of bias (mainly owing to a lack of blinding), with the highest quality studies comparing PRP to hyaluronic acid rather than placebo. ${ }^{9}$ This survey identified that there is a discrepancy between current practice and the evidence base for PRP, especially for tendinopathies. In particular, recent high quality evidence that PRP is not effective (compared to placebo) for lateral epicondylosis appears to have had a limited effect on current beliefs and practice.

While we were quite satisfied with the response and completion rates in this survey, there are some limitations worth pointing out. We relied on the accuracy of the ACSP database in order to ensure completeness of survey invites to Fellows. Nonetheless, we believe a representative sample of responses has been obtained. Additionally, there may have been some oversights in relation to the survey design because of the many permutations of PRP preparation and administration. Participants were not specifically asked about their use of autologous blood injections (as distinct from PRP), or double spin procedures. Yet it is unlikely that participants would not be able to comment on these issues if present, and in fact this further highlights the issue of heterogeneity for this intervention.

There is no consensus on whether PRP should be used, and in this survey there were some strong advocates against PRP within the professional body, who suggested it should be rejected as an intervention altogether. Clinicians raised concerns around the heterogeneity of the procedure without the ability for standardisation, and the lack of research evidence. This study demonstrates that the threshold, indications, preparation and administration of PRP use differ widely among SEM physicians. The lack of standardisation in methodology for PRP preparation creates uncertainty in the generalisability of research conclusions-perhaps perpetuating 
the beliefs that PRP may still be effective when applied with as yet unknown, 'optimal' methodology.

This is the first cross-sectional study of a group of musculoskeletal clinicians to assess practice in relation to PRP use. Previous surveys have assessed the use of cortisone injections among orthopaedic surgeons and found surprising evidence-practice gaps. ${ }^{18}$ The Australasian College of Sports Physicians is a common training programme for specialists working in Australia or New Zealand. Yet we found that Australian sports physicians used PRP much more than New Zealand sports physicians, suggesting that the delivery of this treatment is highly sensitive to healthcare system support. At the time of this survey in 2014, there was generous Medicare Benefits Schedule status for the injection of autologous blood (Item 13 703), providing \$A101.60 for the service as a rebate. ${ }^{19}$ However, at this time in New Zealand, there was no specific remuneration provided to patients or doctors to facilitate this service. As of January 2015, there is no longer a Medicare Item Number for PRP injections, and the treatment no longer attracts a Medicare benefit for patients in Australia. PRP is clearly a divisive issue among sports physicians, and with the jury still out on whether PRP works, the healthcare context will have more influence on the decision to use it than high quality evidence.

\section{CONCLUSIONS}

Even after the withdrawal of Medicare support for the service, PRP will continue to be used as an adjunctive therapy in SEM. Despite its popularity with high-profile athletes, celebrities and cosmetic clinicians in recent years, the best evidence of effectiveness of PRP is for mild-to-moderate severity osteoarthritis (of the knee in particular). Certainly the use of PRP will continue to be a divisive issue among sports physicians, in an environment where clinical evidence conflicts with enthusiasm. Since the disqualification of PRP from Medicare support in Australia, it will be informative to reassess how the change in remuneration for the procedure affects practice among Australasian SEM physicians.

\section{Twitter Follow David Samra at @dave_samra}

Contributors DJS conceived the study. DJS and JWO initiated the study design. DJS conducted the primary statistical analysis. All authors contributed to refinement of the study protocol and approved the final manuscript.

Competing interests None declared.

Ethics approval Sydney University HREC.

Provenance and peer review Not commissioned; internally peer reviewed.

Data sharing statement Unpublished data within the complete data set are available to the researchers for comparison to repeat survey data in 2015. Until this is complete, data will be available only on request.
Open Access This is an Open Access article distributed in accordance with the Creative Commons Attribution Non Commercial (CC BY-NC 4.0) license, which permits others to distribute, remix, adapt, build upon this work noncommercially, and license their derivative works on different terms, provided the original work is properly cited and the use is non-commercial. See: http:// creativecommons.org/licenses/by-nc/4.0/

\section{REFERENCES}

1. Hsu WK, Mishra A, Rodeo SR, et al. Platelet-rich plasma in orthopaedic applications: evidence-based recommendations for treatment. J Am Acad Orthop Surg 2013;21:739-48.

2. Paoloni J, De Vos RJ, Hamilton B, et al. Platelet-rich plasma treatment for ligament and tendon injuries. Clin J Sport Med 2011;21:37-45.

3. Laudy AB, Bakker EW, Rekers M, et al. Efficacy of platelet-rich plasma injections in osteoarthritis of the knee: a systematic review and meta-analysis. Br J Sports Med 2015;49:657-72.

4. Perez AG, Lana JF, Rodrigues AA, et al. Relevant aspects of centrifugation step in the preparation of platelet-rich plasma. ISRN Hematol 2014;2014:176060.

5. Moraes VY, Lenza M, Tamaoki MJ, et al. Platelet-rich therapies for musculoskeletal soft tissue injuries. Cochrane Database Syst Rev 2014;(4):CD010071.

6. Fitzpatrick J, ed. PRP kit validation study. Partners in performance. Wellington, New Zealand: Australasian College of Sports Physicians, 2013.

7. Lee KS, Wilson JJ, Rabago DP, et al. Musculoskeletal applications of platelet-rich plasma: fad or future? AJR Am J Roentgenol 2011;196:628-36.

8. Patel S, Dhillon MS, Aggarwal S, et al. Treatment with platelet-rich plasma is more effective than placebo for knee osteoarthritis: a prospective, double-blind, randomized trial. Am J Sports Med 2013;41:356-64.

9. Sánchez M, Fiz N, Azofra J, et al. A randomized clinical trial evaluating plasma rich in growth factors (PRGF-Endoret) versus hyaluronic acid in the short-term treatment of symptomatic knee osteoarthritis. Arthroscopy 2012;28:1070-8.

10. Beitzel K, Allen D, Apostolakos J, et al. US definitions, current use and FDA stance on use of platelet-rich plasma in sports medicine. J Knee Surg 2015;28:29-34.

11. Team APW. The Australian drug regulatory system [updated 2015/ 01/10/03:58:23]. http://www.australianprescriber.com/magazine/18/3/ 69/71files/621/71.htm

12. Mazzocca AD, McCarthy MB, Chowaniec DM, et al. Platelet-rich plasma differs according to preparation method and human variability. J Bone Joint Surg Am 2012;94:308-16.

13. Dragoo JL, Wasterlain AS, Braun $\mathrm{HJ}$, et al. Platelet-rich plasma as a treatment for patellar tendinopathy: a double-blind, randomized controlled trial. Am J Sports Med 2014;42:610-18.

14. de Vos RJ, Weir A, van Schie HT, et al. Platelet-rich plasma injection for chronic Achilles tendinopathy: a randomized controlled trial. JAMA 2010;303:144-9.

15. de Vos RJ, Windt J, Weir A. Strong evidence against platelet-rich plasma injections for chronic lateral epicondylar tendinopathy: a systematic review. Br J Sports Med 2014;48:952-6.

16. Dean BJ, Lostis E, Oakley T, et al. The risks and benefits of glucocorticoid treatment for tendinopathy: a systematic review of the effects of local glucocorticoid on tendon. Semin Arthritis Rheum 2014;43:570-6.

17. Campbell KA, Saltzman BM, Mascarenhas R, et al. Does Intra-articular Platelet-Rich Plasma Injection Provide Clinically Superior Outcomes Compared With Other Therapies in the Treatment of Knee Osteoarthritis? A Systematic Review of Overlapping Meta-analyses. Arthroscopy 2015.

18. Titchener AG, Booker SJ, Bhamber NS, et al. Corticosteroid and platelet-rich plasma injection therapy in tennis elbow (lateral epicondylalgia): a survey of current UK specialist practice and a call for clinical guidelines. Br J Sports Med 2015;49:1410-13.

19. Services DoHaA. The August 2014 Medicare Benefits Schedule [updated 3 October 2014]. Available from: http://www.mbsonline.gov. au/internet/mbsonline/publishing.nsf/Content/Downloads-201408. 